\title{
Improving Self-Monitoring of Blood Glucose among Adults with Type 1 Diabetes: Results of the Mobile ${ }^{\mathrm{TM}}$ Study
}

Jane Overland $\cdot$ Jessie Abousleiman $\cdot$ Andriana Chronopoulos $\cdot$ Natasha Leader $\cdot$

Lynda Molyneaux $\cdot$ Christopher Gilfillan

To view enhanced content go to www.diabetestherapy-open.com

Received: October 30, 2014 / Published online: December 11, 2014

(c) The Author(s) 2014. This article is published with open access at Springerlink.com

\section{ABSTRACT}

Introduction: The objective of this study was to determine whether people with type 1 diabetes are more likely to self-monitor their blood glucose (SMBG) as recommended by their diabetes health care professional using the Accu-Chek Mobile ${ }^{\mathrm{TM}}$ (F. Hoffmann-La Roche AG, Basel, Switzerland) monitoring system compared to the Freestyle Optium ${ }^{\mathrm{TM}}$ (Abbott, North Chicago, IL, USA).

Trial registration Australian and New Zealand Clinical Trials Registry: \#ACTRN12612000144819.

Electronic supplementary material The online version of this article (doi:10.1007/s13300-014-0092-9) contains supplementary material, which is available to authorized users.

J. Overland · J. Abousleiman · N. Leader .

L. Molyneaux

Diabetes Centre, Royal Prince Alfred Diabetes

Centre, Camperdown, NSW, Australia

J. Overland

The School of Nursing, Sydney, NSW, Australia

A. Chronopoulos · C. Gilfillan ( $₫)$

Eastern Clinical School, Eastern Clinical Research

Unit, Monash University, and Eastern Health,

Box Hill, Melbourne, VIC, Australia

e-mail: chris.gilfillan@easternhealth.org.au
Methods: Thirty-five participants with type 1 diabetes participating in a randomized crossover study were assigned to monitor their blood glucose levels for a 3-month period using the Accu-Chek Mobile or the Freestyle Optium monitoring system and then to cross-over to the alternative device. After completion of the 6-month cross-over period, participants were invited to select their meter of choice and were followed for a further 3 months.

Results: SMBG frequency increased in both groups but participants monitored significantly more often using the Accu-Chek Mobile meter (frequency SMBG/week median: 19 vs. $10, P=0.04)$. After 3 months using each meter, $77 \%$ of participants indicated a preference for the Accu-Chek Mobile meter. Monitoring frequency in this group remained higher than baseline during the 3-month postcross-over follow-up period.

Conclusion: Our results indicate that the AccuChek Mobile meter improves SMBG frequency. After experience of both systems, Accu-Chek Mobile was the meter of choice for the majority of participants in this study.

Funding: Roche Diabetes Care Unconditional Education Grant. 
Keywords: Blood glucose; Compliance; Meter; Satisfaction; Self-monitoring; Type 1 diabetes mellitus

\section{INTRODUCTION}

The findings of the Diabetes Control and Complications Trial (DCCT) and Epidemiology of Diabetes Interventions and Complications (EDIC) studies have highlighted the importance of maintaining glycemic control as close to target as possible in people with type 1 diabetes $[1,2]$. However, such optimization is often difficult to achieve. While intensive treatment of type 1 diabetes has been made possible by combining multiple daily injections (MDI) or continuous subcutaneous insulin infusion (CSII) with frequent self-monitoring of blood glucose (SMBG), many people with type 1 diabetes only measure pre-meal blood glucose levels, making decisions regarding insulin therapy optimization difficult.

There are a number of reasons why people with type 1 diabetes monitor less frequently than recommended. For example, forgetfulness, difficulties with handling and disposing of test strips, lifestyle alignment (embarrassment of monitoring in public, lack of time, and difficulty monitoring away from home), as well as the inability to make decisions based on the results, have all been identified as major barriers to appropriate SMBG [3-5]. To address these issues, a novel, strip-free blood glucose monitoring system has been developed, the Accu-Chek Mobile ${ }^{\mathrm{TM}}$ (F. Hoffmann-La Roche AG, Basel, Switzerland). The Accu-Chek Mobile incorporates a number of key features which eliminate the need to handle test strips or single lancets before and after use, giving the user more freedom and flexibility when monitoring blood glucose, as well as allowing more discrete monitoring. The Accu-Chek Mobile device and other similar integrated devices are estimated to require $70 \%$ fewer steps in the performance of SMBG and previous authors have speculated that this is likely to improve SMBG compliance [6]. The Accu-Chek Mobile device has also performed well in patients' hands, easily reaching an international standard of accuracy [7]. The Accu-Chek Mobile also allows the user to set up to 10 reminder times; at each set time a beep sounds and the reminder time is displayed. A Danish survey of people with type 1 diabetes found the Accu-Chek Mobile system improved adherence to the recommended SMBG frequency [3]. However, to date, there have been no randomized-controlled trials to determine whether the Accu-Chek Mobile system is superior to other currently available monitoring systems.

This randomized-controlled cross-over study was designed to determine whether people with type 1 diabetes are more likely to monitor their diabetes as recommended by their diabetes health care professional using the Accu-Chek Mobile monitoring system compared to the Freestyle Optium $^{\mathrm{TM}}$ (Abbott, North Chicago, IL, USA), a system commonly used by people with type 1 diabetes living in Australia. The study also assessed the effect of the two monitoring systems on peoples' glycemic control, diabetes treatment satisfaction, confidence in treating diabetes, and diabetes distress.

\section{METHODS}

\section{Study Design}

This was a two-center, prospective, randomized cross-over study conducted in two large diabetes units on the Australian Eastern 
seaboard: the Royal Prince Alfred Hospital in Sydney, and Eastern Health in Melbourne. All procedures followed were in accordance with the ethical standards of the responsible committee on human experimentation (institutional and national) and with the Helsinki Declaration of 1975 , as revised in 2000 and 2008. Informed consent was obtained from all patients for being included in the study after adequate explanation of the aims, methods, anticipated benefits, and potential hazards of the study.

Subjects were eligible to participate if they were aged between 18 and 45 years, had type 1 diabetes diagnosed for one or more years, and had a glycosylated hemoglobin $(\mathrm{HbA} 1 \mathrm{c}) \geq 7.5 \%$. Subjects were also required to be monitoring less frequently than recommended by their health care professional (i.e., monitoring on average fewer than three times per day). Participants were excluded if they had conditions that may influence blood glucose monitoring behavior, such as pregnancy or use of steroids.

After signing informed consent, subjects were randomly assigned to one of two possible monitoring sequences: (1) use of the Accu-Chek Mobile system during the first 3 months of the study, crossing over to use the Freestyle Optium system during the second 3 months of the study or, (2) use of the Freestyle Optium system during the first 3 months, crossing over to use the Accu-Chek Mobile system during the second 3 months of the study. The primary outcome of the study was frequency of SMBG per week. Secondary outcomes included glycemic control, satisfaction with treatment, confidence in treating diabetes, and diabetes distress, as well as meter preference.

Participants were required to attend three study visits: baseline (Visit 1), 3 months (Visit 2), and 6 months (Visit 3). Where possible, clinic and research visits were aligned to minimize the participant's attendance to the study sites. At Visits 1 and 2 (baseline and 3 months) the participants were instructed on the use of their assigned meter and they were provided with sufficient supplies to monitor their blood glucose levels up to eight times a day for the following 3 months.

From Visit 2 onwards, the participant's blood glucose monitoring system (Accu-Chek Mobile or Freestyle Optium) memory was downloaded for assessment of monitoring frequency. The memory capacity of each system is 500 and 450 measurements, respectively. Monitoring frequency was calculated as (number of results on the study meter/days of memory) $\times 7$.

Glycemic control was assessed using HbA1c. HbA1c measured at the closest clinical appointment was recorded at each study visit. If the HbA1c had been measured more than 2 weeks prior to study visit, a finger prick sample of blood was collected and an HbA1c estimation was performed using a point of care device (DCA Vantage ${ }^{\circledR}$ Analyzer, Siemens AG, Germany). Participants also completed a series of validated questionnaires at enrolment. These included the Diabetes Treatment Satisfaction Questionnaire (DTSQ), status and change measures (used under license) [4, 5], the Confidence in Diabetes Self-Care (CIDS) scale [8], and the Problem Areas in Diabetes Management (PAID) scale [9].

At the completion of the cross-over study participants were asked to select their study meter of choice. They were also invited to participate in a 3-month extension study. Participants who chose to be involved in this extension study were provided with sufficient supplies to monitor their blood glucose levels up to eight times a day for the following 3 months using their preferred meter. They attended a fourth study visit scheduled 
9 months after their baseline visit and further outcome data were collected as described above.

\section{Data Analysis and Sample Size}

The primary outcome was change in frequency of monitoring from baseline. Using a two-sided one-sample $t$ test $(\alpha=0.05)$, and assuming a drop-out rate of $20 \%$, a sample size of 23 was determined to have $90 \%$ power to detect a difference of at least 4 assessments/week with an estimated standard deviation of 1 .

Data were analyzed using the Number Cruncher Statistical System (NCSS, LLC, Kaysville, UT, USA) software. Each subject acted as their own control; therefore, statistical methods for paired data analysis including paired $t$ test, Wilcoxon signed-rank test, and repeated measures analysis of variance were adopted. Results were regarded as significant at the $P<0.05$ level (two-tailed).

\section{RESULTS}

Forty people with type 1 diabetes were recruited for the study; of these, five were lost to followup. As shown in Table 1, the participants who were lost to follow-up were younger than their counterparts who completed the study. There were no other differences in baseline profiles.

Monitoring frequency increased during both of the 3-month cross-over periods but participants monitored significantly more often during the 3 months they were randomized to use the Accu-Chek Mobile meter (Table 2). There was no difference in participants' satisfaction or confidence in managing diabetes using either monitoring system. The choice of meter also did not impact on the participants' emotional adjustment to living with diabetes as assessed by the PAID scale. After 3 months of using both monitoring systems, the majority of participants $(77 \%)$ indicated a preference for the Accu-Chek Mobile meter.

As described previously, at completion of the 6-month randomized cross-over phase, all participants were invited to be followed in a 3-month extension study. Only two participants did not continue into this extension phase: one withdrew due to pregnancy and the other withdrew as their work required them to move inter-state. The monitoring frequency of the participants who indicated a preference for the Accu-Chek Mobile meter $(n=25)$ remained significantly higher during the 3-month extension phase than at baseline [median 17 [interquartile range (IQR) 8-29] SMBG estimates/week vs. median: 7 (IQR: 3-16) SMBG estimates/week; $P=0.0002]$. These participants also had a small but statistically significant improvement in their glycemic control [average HbA1c: $8.7 \pm 1.1 \%$ $(70.1 \pm 11.3 \mathrm{mmol} / \mathrm{mol})$ at 9 months vs. $9.1 \pm 1.2 \% \quad(75.3 \pm 13.3 \mathrm{mmol} / \mathrm{mol}) \quad$ at baseline; $P=0.04]$. There was no change in monitoring frequency or glycemic control for those participants who indicated a preference for the Freestyle Optium meter $(n=8)$; however, these participants were monitoring more frequently at baseline (median 18 [IQR: 12-28] SMBG estimates/week). They also had a lower baseline average HbA1c $[8.6 \pm 1.1 \%$ $(70 \pm 15.1 \mathrm{mmol} / \mathrm{mol})]$.

\section{DISCUSSION}

The DCCT [1, 2] and EDIC [1, 2] trials demonstrated that intensive glycemic control in type 1 diabetes led to a reduced incidence of microvascular and macrovascular complications in the long term. An essential component of 
Table 1 Baseline demographic and clinical profile for participants completing the study compared to those lost to follow-up

\begin{tabular}{lll}
\hline Characteristic & Lost to follow-up $(\boldsymbol{N}=\mathbf{5})$ & Completed the study $(\boldsymbol{N}=\mathbf{3 5})$ \\
\hline Age (years) & $22.8(1.8)$ & $32.7(10.3)$ \\
Duration (years) $^{\mathrm{b}}$ & $13(6-18)$ & $18(7-25)$ \\
Males (\%) & 60 & 43 \\
Frequency of SMBG per week $^{\mathrm{c}}$ & $6(3-8)$ & $10(3-19)$ \\
HbAlc (\%) & $8.9(2.2)$ & $9.1(1.4)$ \\
HbAlc (mmol/mol) & $76.6(24.3)$ & $75.8(15.4)$ \\
Sum CIDS score & $87.2(12.8)$ & $86.5(13.4)$ \\
CIDS score & $68.6(10.0-78.9)$ & $54.3(22.9-74.3)$ \\
PAID score & $24.4(5.9)$ & $27.6(19.4)$ \\
DTSQ score & $26.2(6.8)$ & $26.1(6.2)$ \\
\hline
\end{tabular}

CIDS confidence in diabetes self-care scale, DTSQ diabetes treatment satisfaction questionnaire, $H b A 1 c$ glycosylated hemoglobin, $P A I D$ problem areas in diabetes management, $S M B G$ self-monitoring of blood glucose

Results expressed as mean (SD) unless otherwise indicated

${ }^{\text {a }} t=2.1, P=0.04$

${ }^{\mathrm{b}}$ Median (range) or ${ }^{\mathrm{c}}$ median (IQR)

intensified insulin therapy, whether by MDI or CSII, is regular SMBG to enable accurate adjustment of insulin to correct both fasting and post-prandial glucose levels. Despite this, compliance with SMBG is poor with up to $24 \%$ of persons with type 1 diabetes testing less than once weekly. Predictors of poor compliance include male gender, younger age, living alone, low diabetes-related concern, and cigarette smoking [10]. High frequency of monitoring has been associated with lower HbA1c and reduced incidence of hypoglycemia, although in some studies recent hypoglycemia was associated with increased monitoring frequency [10-12]. In a study by Schütt et al. [12], HbA1c was reduced by $0.32 \%$ per extra test done per day in people with type 1 diabetes on 4 injections per day or CSII, similar to the participants in our study. One study has related SMBG to long-term complications and has suggested that higher frequencies of monitoring are associated with reduced HbA1c variability and reduced microvascular complications [13].

Other barriers to SMBG include invasiveness. In a study examining the impact of the person's perception of the invasiveness of the SMBG technique [14], 63\% of respondents reported invasiveness was a reason for skipping tests. A semi-quantitative measure of perceived invasiveness correlated negatively with testing frequency [14]. Although there are no published data, the authors were of the opinion that this invasiveness extends beyond the lancet but includes the degree to which the SMBG technique and equipment impact on lifestyle and cause embarrassment through being socially visible. The Accu-Chek Mobile system used in this study has potential advantages in having a connected lancet device and a stripless system utilizing a flexible strip contained within a cartridge. These features make the device potentially simpler to use and removed the need to deal with waste strips. Our 
Table 2 Monitoring frequency (SMBG/week), glycemic control (HbAlc), satisfaction with treatment (DTSQ), confidence in treating diabetes (CIDS score), and diabetes distress (PAID score) at baseline and during the 3-month cross-over periods that participants were randomized to use the Accu-Chek Mobile ${ }^{\mathrm{TM}}$ meter vs. the Freestyle Optium ${ }^{\mathrm{TM}}$ meter

\begin{tabular}{|c|c|c|c|}
\hline Characteristic & Baseline $N=35$ & Accu-Chek Mobile ${ }^{\mathrm{TM}} N=35$ & Freestyle Optium $^{\mathrm{TM}} N=35$ \\
\hline Frequency SMBG/week ${ }^{\mathrm{a}, \mathrm{b}}$ & $10(3-19)$ & $19(9-25)$ & $12(7-26)$ \\
\hline HbAlc (\%) & $9.1(1.4)$ & $8.9(1.3)$ & $8.7(1.3)$ \\
\hline $\mathrm{HbAlc}(\mathrm{mmol} / \mathrm{mol})$ & $75.7(15.4)$ & $72.8(13.3)$ & $71.8(13.2)$ \\
\hline DTSQs & $26.1(6.2)$ & $26.2(6.8)$ & $28.4(6.7)$ \\
\hline $\mathrm{DTSQc}^{\mathrm{a}}$ & - & $10(0-15)$ & $10(-2-14)$ \\
\hline CIDS score & $86.5(13.4)$ & $87.0(9.9)$ & $86.3(11.6)$ \\
\hline PAID score & $27.6(19.4)$ & $24.6(17.5)$ & $29.3(21.7)$ \\
\hline
\end{tabular}

CIDS confidence in diabetes self-care scale, DTSQs diabetes treatment satisfaction questionnaire (status version), DTSQc diabetes treatment satisfaction questionnaire (change version), HbAlc glycosylated hemoglobin, PAID problem areas in diabetes management, $S M B G$ self-monitoring of blood glucose

Results expressed as mean (SD) or ${ }^{\text {a }}$ median (IQR)

${ }^{\mathrm{b}} F=6.2, P=0.003$

comparator, the Freestyle Optium system, was amongst the most popular meters at the time in Australia [15] and uses a conventional blood glucose testing strip that needs to be inserted in the device and disposed of afterwards.

Approaches to increase monitoring frequency have included addressing access and cost issues [16] and some have suggested the importance of linguistically and culturally appropriate education [17]. A 12-week behavioral change program consisting of a course on self-control behavior techniques was effective in increasing SMBG frequency but did not result in achieved SMBG goals [18]. The sustainability of such techniques has not been tested. Meter features have been examined as a determinant of SMBG frequency. In one study the education of people to use the advanced features of a meter, including an audible reminder, increased the frequency of SMBG [19]. With regards to the Accu-Chek Mobile meter system, one previous study has demonstrated an increased compliance with recommended SMBG frequency [3]. In this study of over 1,000 non-compliant patients with type 1 diabetes, 3 months of Accu-Chek Mobile use increased testing frequency from 8.2 tests per week to 18.4 tests per week (goal: 21 tests per week) [3]; this was, however, an uncontrolled and non-randomized study.

We designed a study to examine the effect of two blood glucose monitoring systems on SHBM frequency in a prospective, randomized cross-over study. The primary outcome of the study was the number of SMBG estimations per week. Secondary outcomes included glycemic control, satisfaction with treatment, confidence in treating diabetes, and diabetes distress. Meter preference was assessed at the end of the 6-month cross-over study by allowing the subjects to choose the meter they would use for the final 3 months of the study. The advantages of the design were that subjects were able to experience each of the meters in turn and then express a preference, while the cross-over design ensured that any effect for the order of exposure was controlled for by the randomization. 
Our results indicated that, while all subjects increased their monitoring frequency, persons using the Accu-Chek Mobile system tested more frequently than those using the Freestyle Optium system, and that the majority of patients preferred the Accu-Chek Mobile system. There was no effect of SMBG system on satisfaction and confidence in diabetes treatment or in diabetes-related distress. It is likely, therefore, that the change in SMBG frequency was due to ease of use of the device rather than any change in attitude or more general compliance within the cohort.

Seventy-seven percent of subjects chose to use the Accu-Chek Mobile meter during the 3-month extension period. At the end of 9 months, these subjects had a lower average HbA1c (9.1\% vs. 8.7\%) although they will have used the Freestyle Optium meter for 3 months of the total period. Subjects that chose the Freestyle Optium meter had lower HbA1c at baseline but the numbers in this group were too small to make meaningful comment on the effect of this meter choice on HbA1c over 9 months. Although HbA1c improved during the 3-month cross-over phase of the study there were no significant differences between groups.

Potential sources of bias in our study include previous exposure to one or other of the meters. Subjects came into the study using a variety of meters and some will have been using the Freestyle Optium meter. None were using the Accu-Chek Mobile meter. It is possible that some will have preferred the Accu-Chek Mobile meter simply because of its newness, although the cross-over design helps to mitigate against this. Necessarily, our study was unblinded to both subject and investigator. Investigators took care to avoid expressing bias to one or other meter and all study documentation treated the meters as equal comparators.

\section{CONCLUSION}

The Accu-Chek Mobile blood glucose monitoring system confers an advantage over a popular comparator system through increasing the frequency of SMBG. Although not demonstrated in this study, this has potential benefits for glycemic control and reducing long-term complications.

\section{ACKNOWLEDGMENTS}

The authors wish to extend thanks to Professor Jane Speight for her advice regarding instrument selection and study design. They are also grateful to Jayne Lehmann who was a member of the study's steering committee. All named authors meet the ICMJE criteria for authorship for this manuscript, take responsibility for the integrity of the work as a whole, and have given final approval to the version to be published. The protocol was designed by the authors (JO, CG). The data were collected by the authors (JA, LM, NL, AC), analyzed by the authors (JO) and the paper written by the authors (JO, CG). Roche Diabetes Care funded the project, including the associated article processing charges, through an untied educational grant. Roche Diabetes Care suggested the project and provided the meters and consumables but had no input into design, execution, data collection, analysis, or paper writing. Roche were allowed to review the paper prior to submission but did not influence content.

Conflict of interest. Prof. Christopher Gilfillan was previously a member of the Roche Accu-Chek Advisory Board and received honorary for participation and support for transport to and from meetings. This period 
overlapped with the beginning of the study. Associate Professor Jane Overland remains a member of the Roche Accu-Chek Advisory Board and received honorary for participation and support for transport to and from meetings. Jessie Abousleiman, Andriana Chronopoulos, Natasha Leader, and Lynda Molyneaux declare no competing interests.

Compliance with ethics guidelines. The project received approval from the Human Research Ethics Committees of both Eastern Health and the Royal Prince Alfred Hospital. All procedures were in accordance with the ethical standards of the responsible committee on human experimentation (institutional and national) and with the Helsinki Declaration of 1975, as revised in 2000 and 2008. Informed consent was obtained from all patients for being included in the study.

Open Access. This article is distributed under the terms of the Creative Commons Attribution Noncommercial License which permits any noncommercial use, distribution, and reproduction in any medium, provided the original author(s) and the source are credited.

\section{REFERENCES}

1. The Diabetes Control and Complications Trial Research Group. The effect of intensive treatment of diabetes on the development and progression of long-term complications in insulin-dependent diabetes mellitus. N Engl J Med. 1993;329:977-86.

2. Nathan DM, Cleary PA, Backlund JY, et al. Intensive diabetes treatment and cardiovascular disease in patients with type 1 diabetes. $\mathrm{N}$ Engl J Med. 2005;353:2643-53.

3. Mast O, Schmidt BA (2010) Using a strip-free system for self-monitoring of blood glucose (SMBG) resulted in increased test frequencies in nonadherent testers on flexible insulin therapy-a user survey. Tenth Annual Diabetes Technology Meeting. Bethesda, Maryland, USA.

4. Bradley C. Diabetes treatment satisfaction questionnaire. Change version for use alongside status version provides appropriate solution where ceiling effects occur. Diabetes Care. 1999;22:530-2.

5. Bradley C, Lewis KS. Measures of psychological well-being and treatment satisfaction developed from the responses of people with tablet-treated diabetes. Diabet Med. 1990;7:445-51.

6. Freckmann G, Schmid C, Ruhland K, Baumstark A, Haug C. Integrated self-monitoring of blood glucose system: handling step analysis. J Diabetes Sci Technol. 2012;6:938-46.

7. Sachse D, Bolstad N, Jonsson M, et al. The AccuChek Mobile blood glucose monitoring system used under controlled conditions meets ISO 15197 standards in the hands of diabetes patients. Scand J Clin Lab Invest. 2012;72:374-9.

8. Van Der Ven NC, Weinger $\mathrm{K}, \mathrm{Yi}$ J, et al. The confidence in diabetes self-care scale: psychometric properties of a new measure of diabetes-specific selfefficacy in Dutch and US patients with type 1 diabetes. Diabetes Care. 2003;26:713-8.

9. Welch GW, Jacobson AM, Polonsky WH. The problem areas in diabetes scale. an evaluation of its clinical utility. Diabetes Care. 1997;20:760-6.

10. Hansen MV, et al. Frequency and motives of blood glucose self-monitoring in type 1 diabetes. Diabetes Res Clin Pract. 2009;85(2):183-8.

11. Miller KM, Pedersen-Bjergaard U, Heller SR, et al. Evidence of a strong association between frequency of self-monitoring of blood glucose and hemoglobin A1c levels in T1D exchange clinic registry participants. Diabetes Care. 2013;36:2009-14.

12. Schütt M, Kern W, Krause U, et al. Is the frequency of self-monitoring of blood glucose related to longterm metabolic control? Multicenter analysis including 24,500 patients from 191 centers in Germany and Austria. Exp Clin Endocrinol Diabetes. 2006;114:384-8.

13. Huang IC, Wang PW, Liu RT, et al. The influence of self-monitoring blood glucose frequency on the oscillation of hemoglobin $\mathrm{A} 1 \mathrm{c}$ and chronic complications. Chang Gung Med J. 2012;35:46-53.

14. Wagner J, Malchoff C, Abbott G. Invasiveness as a barrier to self-monitoring of blood glucose in diabetes. Diabetes Technol Ther. 2005;7:612-9.

15. Roche internal company data. 
16. Nyomba BL, Berard L, Murphy LJ. Facilitating access to glucometer reagents increases blood glucose selfmonitoring frequency and improves glycaemic control: a prospective study in insulin-treated diabetic patients. Diabet Med. 2004;21:129-35.

17. Karter AJ, Ferrara A, Darbinian JA, Ackerson LM, Selby JV. Self-monitoring of blood glucose: language and financial barriers in a managed care population with diabetes. Diabetes Care. 2000;23:477-83.

18. Jones PM. Use of a course on self-control behavior techniques to increase adherence to prescribed frequency for self-monitoring blood glucose. Diabetes Edu. 1990;16:296-303.

19. Bergenstal RM, Bode BW, Tamler R, et al. Advanced meter features improve postprandial and paired self-monitoring of blood glucose in individuals with diabetes: results of the actions with the CONTOUR blood glucose meter and behaviors in frequent testers (ACT) study. Diabetes Technol Ther. 2012;14:851-7. 